\title{
QUANTIFYING THE FIR INTERACTION ENHANCEMENT IN PAIRED GALAXIES
}

\author{
Cong Xu \\ International Center for Theoretical Physics, P.O. Box 586, I-34100 Trieste, Italy \\ Jack W. Sulentic \\ University of Alabama, Tuscaloosa 35487 USA
}

\section{Introduction}

We study the "Catalogue of Isolated Pairs of Galaxies in the Northern Hemisphere" (CPG hereafter) by Karachentsev (1972), and a well matched comparison sample taken from the "Catalog of Isolated Galaxies" (Karachentseva 1973, CIG hereafter), in order to quantify the enhanced FIR emission properties of interacting galaxies (see Sulentic 1989 for details of the samples). The isolation criterion used in compiling the CPG and the CIG means that both the pairs and single galaxies have been little influenced by their environment for a long time $\left(t \gtrsim 10^{\circ}\right.$ yrs: Stocke et al 1978).

Some relevent issues addressed in the study are:

1. How populous are isolated pairs? What is the contribution of the paired galaxies to the optical luminsity function of field galaxies?

2. What is the contribution of CPG pairs to the Far-Infrared (IRAS) luminosity function?

3. Does the FIR enhancement of a pair depend on (1) the existance of particular signs of interaction (tails, bridges, etc.) or (2) the separation between the two components?

\section{Optical luminosity functions (OLF's)}

We use the classical estimator of the luminosity function (Felten 1976). The $\left\langle V / V_{m}\right\rangle$ tests demonstrate that neither the pair sample nor the comparison sample is complete at any magnitude level. However, the redshift distributions of the two samples are fairly homogeneous. The incompleteness is corrected using the method suggested by Huchra and Sargent (1973). For CPG samples the correction is about a factor 2. For the comparison sample, it is about a factor of 5 , which also takes into account the incompleteness of the redshift data.

Figure 1 plots the OLF's for individual galaxies in the CIG and in the CPG pairs. CPG pairs are dominant by SS pairs in general. There is a deficiency of early type dwarf galaxies (fainter than -18 magnitude) in EE pairs. The shape of OLF of galaxies in SE pairs is middle way between the OLF's of galaxies in SS pairs and of galaxies in EE pairs. The parameters of the best fitting Schechter function for the OLF of CPG galaxies are $\alpha=-1.2$ and $M_{*}=-20.0$. For CIG galaxies, $\alpha=-1.3$ and $M_{*}=-19.4$. Galaxies in pairs are about 0.6 magnitude brighter than CIG galaxies. Comparing with the OLF of CFA field galaxies (Davis and Huchra 1982), we find that paired galaxies represent about $10 \%$ of field galaxies over the entire luminosity range. 


\section{FIR luminosity functions (FIRLF's)}

The FIR data of both the CPG and the CIG is generally taken from the second version of IRAS Point Source Catalog (PSC). The only exceptions are some galaxies larger than $D=5$ arcmin, for which coadded data are taken from the literature.

Since most pairs in the CPG sample are unresolved by IRAS, we can not derive the FIR luminosity functions for individual CPG galaxies. The FIRLF's for SS pairs and for SE pairs are calculated separately. The FIR luminosity of SE pairs is attributed exclusively to the $S$ component (Paper I). We did not calculate the FIRLF for EE pairs, because the IRAS detection rate is very low $(<11 \%)$. The FIRLF of S/Irr galaxies is derived for the comparison sample.

Because both the pair sample and the comparison sample are optically selected, the FIR luminosity functions are derived from the corresponding optical luminosity functions and the fractional bivariate functions between the FIR and optical luminosities. The correction for incompleteness of the samples affects the FIR luminosity function through the optical luminosity function. In order to take into account the information content of upper limits, the KaplanMeier estimator (Schmitt 1985; Feigelson and Nelson 1985), has been used in computing the bivariate functions and the associated errors.

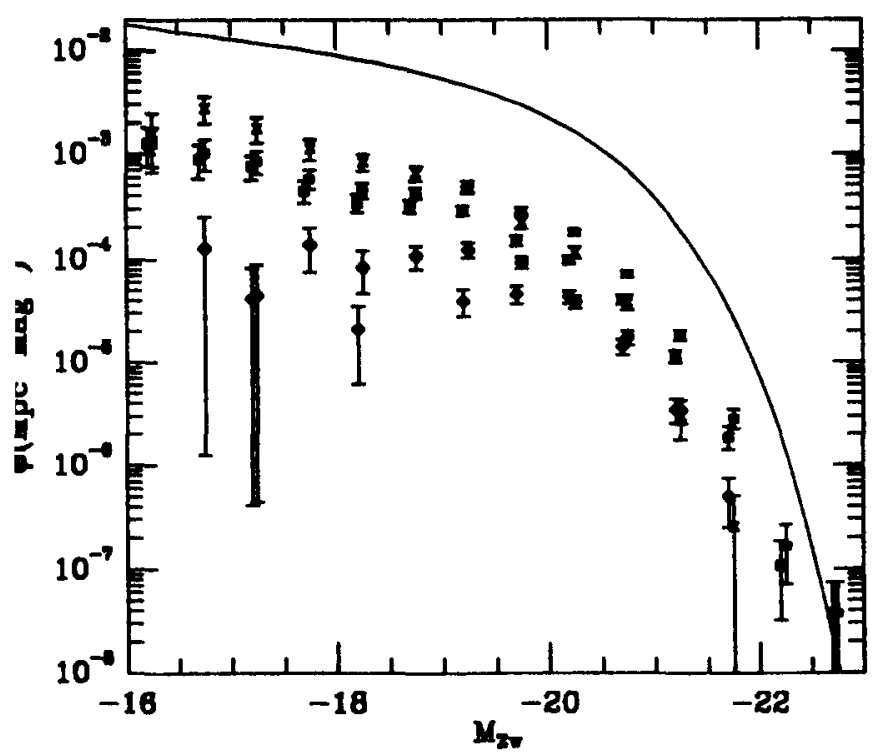

Fig.1 The OLF of CPG galaxies (solid squares), which is decomposed to 3 components: of galaries in SS pairs (open squares), of galaxies in SE pairs (open diamonds), and of galaxies in EE pairs (solid diamonds). The crosses stand for the OLF of CIG galaxies. The solid curve illustrates the OLF of CFA field galaxies (Davis and Huchra 1982).

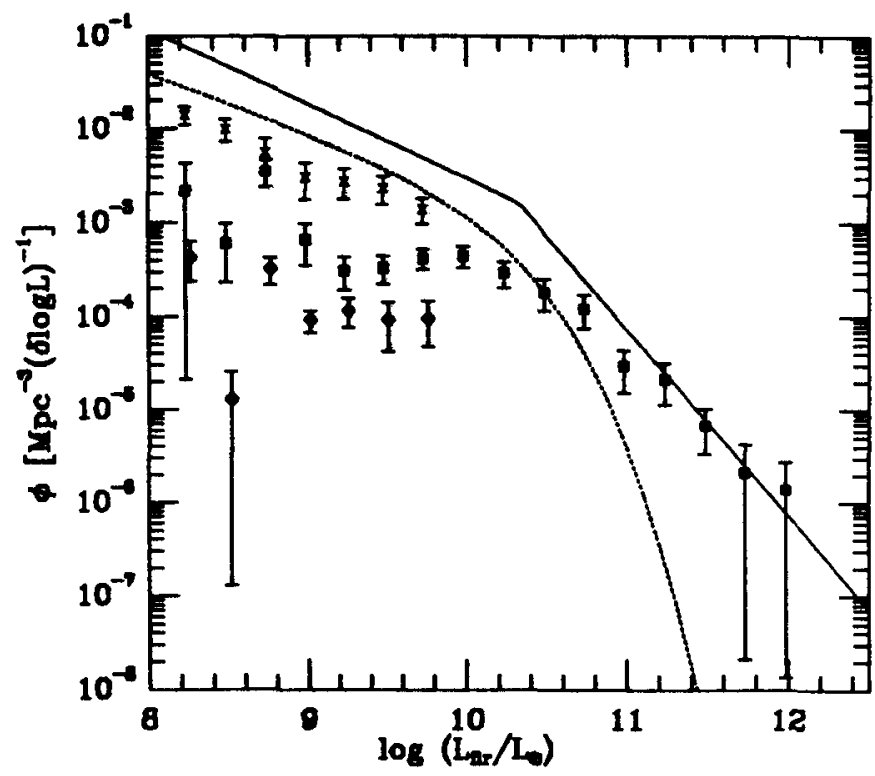

Fig.2 The FIRLF's of of SS pairs (open squares), of CIG S/Irr galaries (crosses), and of SE pairs (open diamonds). The dotted curve represents the FIRLF of the synthetic pairs. The solid curve illustrates the FIRLF of bright IRAS galaxies (Soifer et al. 1987).

FIRLF's are plotted in Fig.2. We divide them into two parts (i.e. above and below $\left.10^{10} L_{\odot}\right)$. In the faint part, the contribution from SS pairs to the IRAS luminosity function is generally less than $10 \%$. In the bright part, the contribution from SS pairs increases with luminosity, becoming dominant beyond $2 \times 10^{11} L_{\odot}$. For isolated S/Irr galaxies the contribution is about $20-30 \%$ in the faint part, and decreases rapidly in the bright part. SE pairs never 
give a significant contribution to the IRAS luminosity function. The mean $\log \left(L_{f i r} / L_{\odot}\right)$ of SS pairs is $9.88 \pm 0.05$. For isolated S/Irr galaxies, it is $9.10 \pm 0.05$. Therefore, SS pairs are about a factor of 6 brighter ( $\sim 10 \sigma$ level) in FIR than isolated S/Irr galaxies on average. The mean $\log \left(L_{f i r} / L_{\odot}\right)$ of SE pairs is $9.52 \pm 0.09$.

For comparison, we have also calculated the FIR luminosity function for a sample of synthetic pairs, which are constructed from isolated S/Irr galaxies. We take into account the fact that CPG pairs are biased to have members of similar optical brightness. The result is represented by the dotted curve. Comparing with it, we find that SS pairs are under-represented in the faint part, and over-represented in the bright part.

\section{The FIR to optical luminosity ratio and the FIR color}

The FIR to optical luminosity ratio and the FIR color (the ratio between the fluxes at 60 microns and at 100 microns) are two important FIR emission indicators. In the folllowing we will concentrate on the SS subsample of CPG and CIG S/Irr galaxies. The FIR properties of SE pairs will be discussed in a separate paper (Sulentic, in preparation).

Upperlimits in FIR data are taken into account in the analyses of the FIR to optical luminosity ratio, and the Kaplan-Meier estimator is used. The cumulative $R=\log L_{f i r} / L_{B}$ distributions of SS pairs and of CIG S/Irr galaxies are different at $10^{-3}$ significance level. The differential $R$ distribution of SS pairs (Fig.3) tiltes reletive to that of isolated S/IR galaxies, in the sense that SS pairs show high $R$ more frequently, and low $R$ less frequently. No evidence of bimodality in the distribution is found. The mean R of SS pair is $-0.16 \pm 0.03$, more than $3 \sigma$ higher than that of isolated spirals, which is $-0.30 \pm 0.03$. The indicated enhancement in the FIR to optical luminoisty ratio is a factor of 1.4 .

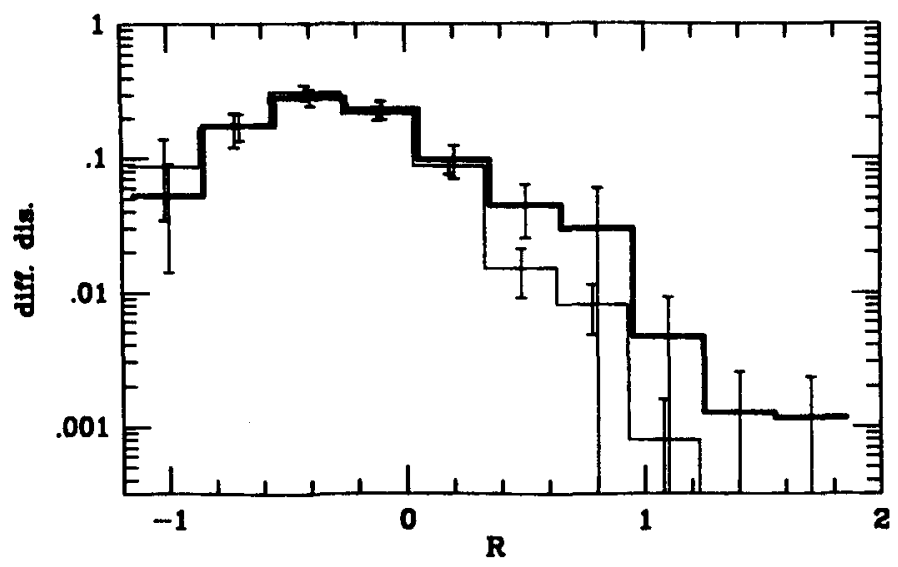

Fig.3 The differential distribution of $R=$ $\log L_{f i r} / L_{B}$. The thick line is the distribution of SS pairs. The thin line is the distribution of isolated S/Irr galaxies.

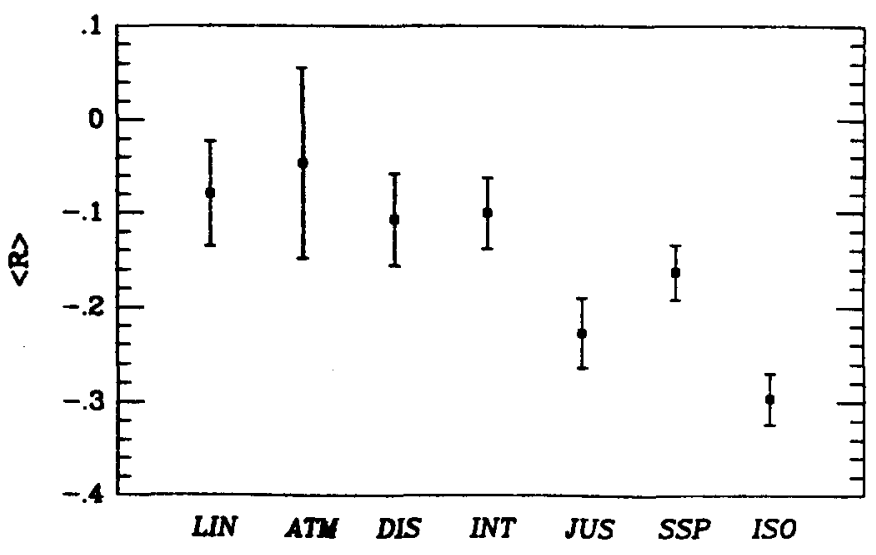

Fig.4 The $<R>$ versus interaction morphology plot (see the text for definitions).

Figure 4 plots the dependence of mean $R$ on the interaction morphology. LIN represents pairs with bridges and/or tails. ATM are pairs in a common luminous envelope. DIS are pairs 
with one or both of the components showing signs of distortion. INT represents interacting pairs on the whole, which is the summary of the above three subclasses. JUS represents pairs without obvious signs of interaction. SSP is the entire sample of SS pairs, which is the combination of INT pairs and JUS pairs. ISO stands for the isolated S/Irr galaxy sample. The $<R>$ of INT pairs is significantly higher than isolated S/Irr galaxies (at $4.2 \sigma$ level) and than JUS pairs (at 2.4 $\sigma$ level). There is a slight enhancement of $\langle R>$ of JUS pairs comparing to isolated S/Irr galaxies (at $1.5 \sigma$ level). There is no significant difference in $\langle R\rangle$ among the three subclasses of interacting pairs.

We define a redshift-independent separation parameter $S E P$ as the ratio between the separation of two components and the size of the primary:

$$
S E P=\frac{\text { component separation (arcmin) }}{\text { size of primary (arcmin) }}
$$

INT pairs with $S E P \leq 1$ demonstrate significantly higher $<R>$ (at $3.2 \sigma$ level) than JUS pairs with $S E P \leq 1$. On the other hand, no difference in $\langle R>$ is found between INT and JUS pairs of $S E T>1$. Moreover, $<R>$ of INT pairs with SEP $\leq 1$ is systematically larger than that of INT pairs with SEP $>1$ (at $2.3 \sigma$ level).

Only detected FIR data are included in the analysis of the FIR color. The cumulative $C=\log F_{60 \mu} / F_{100 \mu}$ distributions of SS pairs and of CIG S/Irr galaxies are different at $<10^{-3}$ significance level. The differential C distribution of SS pairs (Fig.5) shows a systimatical shift toward the bright end comparing to isolated S/IR galaxies. Again no evidence of bimodality in the distribution is found. The mean C of SS pairs is $-0.36 \pm 0.01$, more than $5 \sigma$ higher than that of isolated spirals, which is $-0.42 \pm 0.01$.

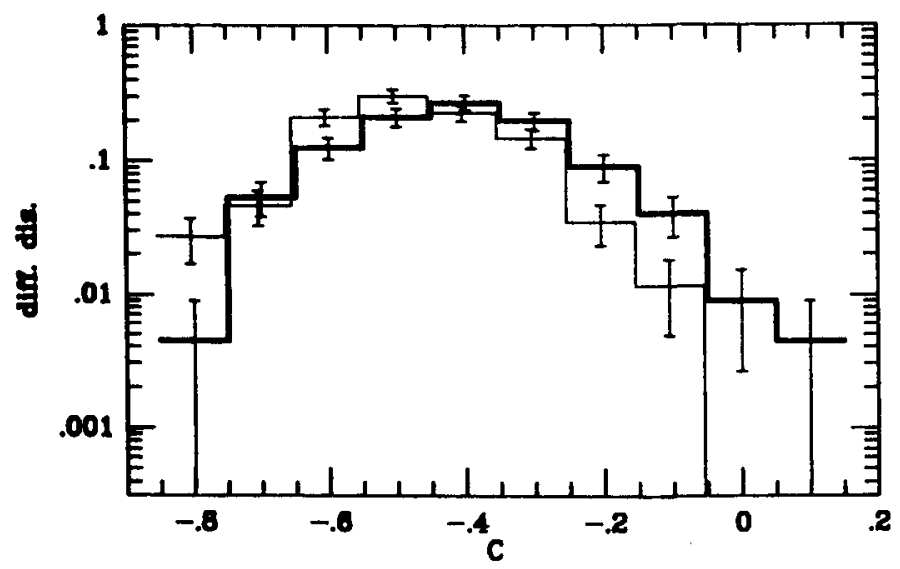

Fig.6 The differential distribution of $C=$ $\log F_{60 \mu} / F_{100 \mu}$. The thick line is the distribution of SS pairs. The thin line is the distribution of isolated S/Irr galaxies.

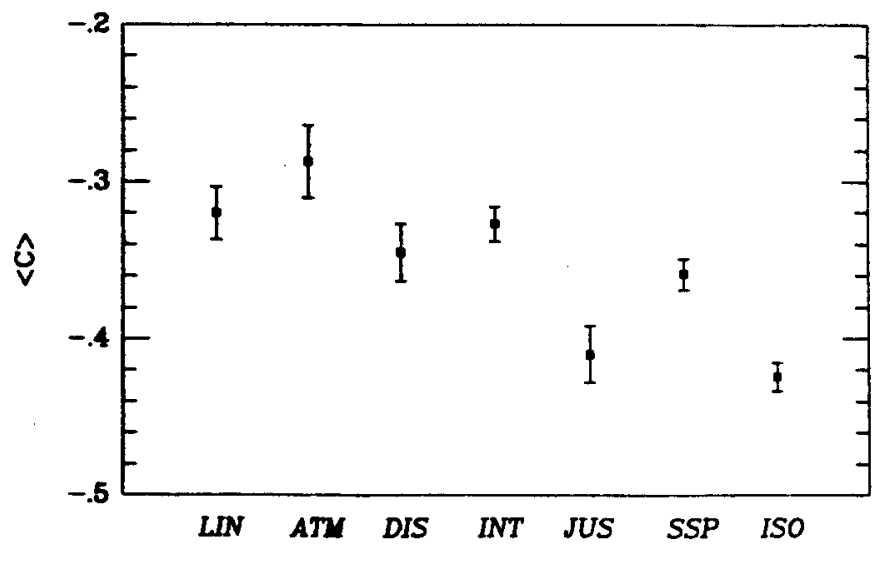

Fig.6 The $\langle C\rangle$ versus interaction morphology plot.

Figure 6 plots the dependence of mean $C$ on the interaction morphology. We find no significant difference between JUS pairs and isolated galaxies, nor among the three subclasses of interacting pairs. The difference between INT pairs and JUS pairs is obvious (at $3.9 \sigma$ level). 
The dependence of $\langle C\rangle$ on SEP is essentially the same with that of $\langle R>$. INT pairs with $S E P \leq 1$ show significantly higher $\langle C\rangle$ than JUS pairs (at $4.6 \sigma$ level) and than INT pairs with SEP> 1 (at $2.6 \sigma$ level).

In summary, it appears that the SS pairs which show signs of interaction, and whose component galaxies are close to each other $(S E P \leq 1)$ demonstrate the most significant enhacement in their mean $R$ and mean $C$ relative to isolated S/Irr galaxies, while other SS pairs show only weak FIR enhancement. We will refer the former as close interacting pairs. In the SS sample, 120 pairs belong to this class, representing $40 \%$ of all (299) SS pairs.

Our results support a bimodal star-formation scenario. The self propagating-regulating mode controls the star-formation in isolated galaxies, while an interaction-stimulated mode should be added to paired galaxies. The amplitude of the later depends on the strength of interaction. For those pairs other than close interacting ones, the effect of the interaction induced star-formation is so weak that it is almost buried by the normal starformation. For close interacting pairs in the SS pair sample, the interaction provides a significant but not ultraluminous effect.

\section{Summary}

(1) About $10 \%$ field galaxies are in isolated pairs.

(2) SS pairs dominate the bright end of the IRAS FIRLF ( $\left.L_{f i r}>2 \times 10^{11} L_{\odot}\right)$, while their contribution to the faint part of the IRAS luminosity function $\left(L_{f i r}<10^{10} L_{\odot}\right)$ is low $(<10 \%)$.

(3) SS pairs on the whole show statistically significant enhancements in their mean FIR to optical luminosity ratio $(\sim 3 \sigma)$ and in their FIR color $(\sim 5 \sigma)$, comparing to isolated S/Irr galaxies.

(4) Detailed study shows that the amplitude of FIR enhancement depends on the intensity of interaction. For the SS pairs which show signs of interaction (tails, bridges, common envelops, distortions, etc.), and whose component galaxies are close to each other ( $S E P \leq$ 1) the enhancement is significant. These "close interacting pairs" represent $\sim 40 \%$ of all SS pairs. For other SS pairs, the enhancement is weak.

\section{References}

Davis, M. and Huchra, J. (1982), Ap.J., 254, 437.

Felten, J. E. (1976), Ap.J., 207, 700.

Feigelson, E. D. and Nelson, P. I. (1985), Ap.J., 283, 192.

Huchra, J. and Sargent, W. L. W. (1973) Ap.J., 186, 433.

Schmitt, J. H. M. M. (1985), Ap.J., 293, 178.

Soifer, B. T., Sanders, D. B., Madore, B. F., Neugebauer, G., Danielson, G. E., Elias, J. H., Lonsdale, C. J. and Rice, W. L. (1987), Ap.J., 320, 238.

Stocke, J., Tifft, W. and Kaftan-Kassim, M. (1978), A.J., 83, 322.

Sulentic, J. (1989), A.J., 98, 2066 (Paper I). 